\title{
The variable phenotype and low-risk nature of RAS-positive thyroid nodules
}

\author{
Marco Medici ${ }^{1}$, Norra Kwong ${ }^{1}$, Trevor E. Angell ${ }^{1}$, Ellen Marqusee ${ }^{1}$, Matthew I. Kim', Mary C. Frates ${ }^{3}$, \\ Carol B. Benson ${ }^{3}$, Edmund S. Cibas ${ }^{2}$, Justine A. Barletta ${ }^{2}$, Jeffrey F. Krane ${ }^{2}$, Daniel T. Ruan ${ }^{4}$, Nancy L. Cho ${ }^{4}$, \\ Atul A. Gawande ${ }^{4}$, Francis D. Moore $\mathrm{Jr}^{4}$ and Erik K. Alexander ${ }^{1 *}$
}

\begin{abstract}
Background: Oncogenic mutations are common in thyroid cancers. While the frequently detected RAS-oncogene mutations have been studied for diagnostic use in cytologically indeterminate thyroid nodules, no investigation has studied such mutations in an unselected population of thyroid nodules. No long-term study of RAS-positive thyroid nodules has been performed.

Methods: We performed a prospective, blinded cohort study in 362 consecutive patients presenting with clinically relevant $(>1 \mathrm{~cm})$ thyroid nodules. Fine needle aspiration cytology and mutational testing were obtained for all nodules. Post-operative histopathology was obtained for malignant or indeterminate nodules, and benign nodules were sonographically followed. Histopathological features were compared between RAS- and BRAF-positive malignancies. RAS-positive benign nodules were analyzed for growth or cellular change from prior aspirations.

Results: Overall, 17 of 362 nodules were RAS-positive. Nine separate nodules were BRAF-positive, of which eight underwent surgery and all proved malignant (100\%). Out of the 17 RAS-positive nodules, ten underwent surgery, of which eight proved malignant (47\%). All RAS-positive malignancies were low risk - all follicular variants of papillary carcinoma, without extrathyroidal extension, metastases, or lymphovascular invasion. RAS-positivity was associated with malignancy in younger patients $(P=0.028)$. Of the nine RAS-positive benign nodules, five had long-term prospective sonographic follow-up (mean 8.3 years) showing no growth or signs of malignancy. Four of these nodules also had previous aspirations (mean 5.8 years prior), all with similar benign results.

Conclusions: While RAS-oncogene mutations increase malignancy risk, these data demonstrate a low-risk phenotype for most RAS-positive cancers. Furthermore, cytologically benign, yet RAS-positive nodules behave in an indolent fashion over years. RAS-positivity alone should therefore not dictate clinical decisions.
\end{abstract}

\section{Background}

Over the last two decades, the discovery of molecular pathways critical to oncogenic transformation has dramatically altered our understanding of thyroid malignancy. Reports initially suggested that nearly $70 \%$ of thyroid cancers harbor single gene mutations in the BRAF or RAS pathways, or balanced translocations of $R E T / P T C$, or PAX8/PPARY [1, 2]. More recently, reports confirm an oncogenic mutation in $97 \%$ of welldifferentiated papillary carcinomas [3]. Such mutations

\footnotetext{
* Correspondence: ekalexander@partners.org

'Thyroid Section, Division of Endocrinology, Hypertension and Diabetes, The Brigham and Women's Hospital and Harvard Medical School, 75 Francis

Street, PBB-B4 Room 417, Boston, MA 02115, USA

Full list of author information is available at the end of the article
}

can increasingly be identified in both preoperative fine needle aspiration (FNA) cytology specimens and on post-operative histopathology. Many believe that the synergistic use of microscopic and molecular analysis is destined to improve the clinical management of this illness [4].

Mutations or translocations identified in most thyroid carcinomas are known to activate pathways regulating cellular growth, development, and/or malignant transformation, which has led to the assumption that thyroid nodules harboring such mutations are either cancerous, or at high risk for eventual malignant transformation [5-7]. However, observational data question this assumption as absolute and suggest that all such pathways or activating mutations may not prove equally oncogenic 
or may require secondary molecular 'hits' before behaving in a malignant fashion. In particular, clinical outcomes of patients with mutations in the $\mathrm{N}-, \mathrm{K}-$, or $\mathrm{H}-$ isoforms of the RAS gene are highly variable [7]. In contrast to homogeneous BRAF V600E mutations which strongly associate with higher-risk papillary carcinoma, initial observations suggest RAS mutations associate with variable histology, ranging from benign disease, to low-risk malignancy, to anaplastic carcinoma [7-10].

However, few prospective blinded assessments of RASpositive thyroid nodules (treated or untreated) have been performed. Such an assessment is critically important, as diagnostic testing for these mutations is now widely available. To many clinicians, identification of an activating $R A S$ mutation may prompt a belief that malignancy has been identified [7]. This leads to downstream clinical recommendations usually favoring surgical resection. Such treatment is highly beneficial in many scenarios, but for those with benign disease, has subjected the patient to unnecessary cost, operative morbidity, and significant risk [11, 12]. Past experience involving papillary microcarcinoma provides a cautionary parallel supporting the importance of further study of $R A S$-positive thyroid nodules. For decades, widespread belief that all papillary carcinoma posed danger prompted recommendations for active removal of such thyroid nodules [13, 14]. This was true even when nodules were smaller than $1 \mathrm{~cm}$ and ultrasound confirmed the absence of abnormal adenopathy. Prospective data debunked this belief, confirming the indolent nature of these papillary microcarcinomas [15]. Expert guidelines now recommend against evaluation of most nodules $<1 \mathrm{~cm}$, and/or surgical treatment of subcentimeter papillary carcinomas $[16,17]$.

We performed a prospective study of clinically relevant thyroid nodules, including ultrasound-guided FNA and gene mutation analysis. Blinded mutational analysis and histopathologic interpretation was performed. Our goal was not to validate the performance of a molecular diagnostic test on cytologically indeterminate nodules, as such investigations have previously been performed. Rather, we uniquely sought to evaluate the molecular profiles of a large consecutive cohort of thyroid nodules $>1 \mathrm{~cm}$ presenting for FNA. By doing so, we sought to understand the molecular profiles detected in an unselected nodule population whether cytologically benign, indeterminate, or malignant. Furthermore, as a large proportion of our patients also participate in an ongoing prospective, long-term cohort study assessing the natural history of thyroid nodules, we hypothesized that several mutation-positive nodules would also have longterm sonographic follow-up for analysis. If so, assessment for potential malignant transformation - in particular when observing the natural history of RAS-positive thyroid nodules - would be for the first time possible.

\section{Methods}

We performed a prospective, blinded study of euthyroid patients seeking care of thyroid nodules $>1 \mathrm{~cm}$ in diameter. Patients were referred to the thyroid biopsy clinic at the Brigham and Women's Hospital (Boston, MA, USA) between July 2010 and October 2012, and evaluated according to current clinical practice guidelines [16]. Ultrasound evaluation was performed by one of four radiologists with expertise in thyroid evaluation, using a $6-15 \mathrm{mHz}$ transducer. Following informed consent, ultrasound-guided FNA was performed by one of three thyroidologists. Three needle passes from the same nodule using $25 \mathrm{~g}$ needles were rinsed into a liquidbased solution (CytoLyt ${ }^{\circ}$; Cytyc Corp., Marlborough, MA, USA), constituting a single aspiration. An additional sample was then obtained and shipped to a centralized CLIA-certified laboratory at Asuragen, Inc. (Austin, TX, USA) where mutational analysis was performed as part of the miRInform Thyroid $^{\bullet}$ diagnostic test [18]. As previously described, this test evaluates 17 distinct genetic alterations, including $14 B R A F, K-, N-$, or H-RAS mutations, and three PAX8-PPARY and RET-PTC rearrangements [18]. Of 391 nodules enrolled for initial evaluation, 11 yielded insufficient nucleic acid for mutational testing. A separate consecutive group of 15 separate nodules all showed an uncharacteristic molecular result (double positive for PAX8-PPARG and RAS) suggesting external cross-contamination during processing of this series, and were therefore excluded. Three nodules were excluded as a result of a protocol deviation (one delayed shipping) or study dropout $(\mathrm{n}=2)$. This resulted in a final population of 362 nodules from 318 patients.

FNA cytology was classified according to the Bethesda system for reporting thyroid cytopathology [19]. Benign cytologic results most often prompted a conservative, non-operative recommendation. Cytologically indeterminate and malignant nodules most often prompted a recommendation for surgical resection. Midway through this study, Afirma gene expression classifier (GEC) testing became available, and was applied to a minority of low-risk patients with initial atypia of undetermined significance (AUS) cytology. If Afirma testing was benign, nodules were treated similarly to those with benign cytology [20]. Following thyroidectomy, histopathology interpretation was performed, which frequently involved multi-expert review and consensus. All histopathologic interpretations were blinded to molecular results. Similarly, molecular interpretation was performed without knowledge of any clinical or pathologic findings. At study completion, results were combined and interpreted.

The Brigham and Women's Hospital thyroid nodule clinic has prospectively enrolled all patients evaluated between 1995-present in an ongoing clinical trial assessing 
the natural history of thyroid nodules [21]. We analyzed all past and present thyroid sonographic imaging in patients with $R A S$-positive, cytologically benign thyroid nodules. For some of the patients, a separate ultrasound-guided FNA of the target nodule had also been performed at an earlier time point before enrollment in the current study. This was usually at a separate facility or by a separate provider.

For the purposes of this study, we sought to specifically describe the histologic outcome of all thyroid nodules positive for $\mathrm{N}_{-}, \mathrm{H}-$, and $K-R A S$ mutations, as these mutations are common yet observations suggest a variable phenotype. For RAS-positive nodules confirmed malignant by histopathology, we documented the cancer type and specific papillary thyroid carcinoma (PTC) variant. Nodule size, as well as multifocality, lymphovascular invasion, extrathyroidal extension, and local metastatic adenopathy were similarly documented. For $R A S$-positive nodules with benign cytology, we reviewed all available sonographic and cytologic reports documenting thyroid nodule size, abnormal adenopathy, or other signs of malignant behavior, with a follow-up time of at least 6 months. Growth was defined as a $>20 \%$ change in the largest two nodule dimensions.

This protocol was approved by the Investigational Review Board of the Brigham and Women's Hospital, and all patients provided written informed consent for participation and publication of individual patient data, including the data described in Tables 2 and 4. No patients received a stipend for participating in this study. ANOVA was used to compare the mean age between patients with RAS-positive benign and malignant nodules. Statistical analysis was performed using SPSS version 22 (SPSS IBM, Armonk, NY, USA), and a $P$ value $<0.05$ was considered significant.

\section{Results}

We prospectively enrolled 318 patients with 362 clinically relevant thyroid nodules $(>1 \mathrm{~cm})$, whose baseline characteristics are shown in Table 1. Following blinded molecular analysis, 17 nodules were positive for mutations in the $\mathrm{K}-, \mathrm{N-}$, or $H_{-}$RAS genes, while nine separate nodules harbored V600E BRAF mutations. Three additional nodules were positive for translocations involving the PAX8-PPARY genes, while no RET-PTC translocations were found. Ultimately, 33 of $362(9.1 \%)$ nodules proved malignant following surgical resection and blinded histopathologic assessment, including eight $B R A F$-positive and eight $R A S$-positive nodules. Of the $17 R A S$-positive nodules, ten were referred to surgery because of abnormal or malignant cytology, while seven did not have surgery as their biopsy was benign (six cytologically 'benign'; one nodule cytologically AUS but subsequent 'benign' GEC). Of the ten RAS-positive nodules referred to surgery, eight proved histologically malignant, while two were histologically benign. In summary, 8 of 17 RAS mutation-positive nodules (47\%) were malignant by microscopic analysis, while the remainder were benign.

\section{RAS-positive thyroid malignancies}

Table 2 shows the characteristics of the RAS-positive thyroid malignancies. All $(\mathrm{n}=8)$ were follicular variants of PTC (fvPTC), and all demonstrated very low-risk characteristics. Specifically, there was no evidence of lymphovascular invasion, extrathyroidal extension, local lymph node metastases, or distant metastases in any $R A S$-positive thyroid cancers. Furthermore, all were encapsulated, or partially encapsulated/well-circumscribed malignancies, and there were no disease-specific deaths. For comparison, we evaluated the histologic characteristics of the $B R A F$-positive thyroid malignancies. Compared to the $R A S$-positive malignancies, the $B R A F$-positive malignancies were equal in size $(2.1 \mathrm{~cm})$. However, they had less favorable histological characteristics as shown in Table 3. These data suggest a more indolent phenotype among many $R A S$-positive thyroid cancers.

\section{RAS-positive benign thyroid nodules}

Nine $R A S$-positive thyroid nodules proved benign (two histologically benign; six cytologically benign; one benign GEC). Characteristics of the RAS-positive benign nodules are shown in Table 4. These nodules averaged $2.1 \mathrm{~cm}$ in largest dimension at study entry. While four nodules had not been previously evaluated in our thyroid nodule clinic, five nodules had undergone previous sonographic imaging and evaluation, including four with

Table 1 Baseline characteristics of the study population and the RAS-positive subgroup

\begin{tabular}{|c|c|c|c|c|c|c|c|}
\hline & $\begin{array}{l}\text { Number of } \\
\text { patients }\end{array}$ & $\begin{array}{l}\text { Number of } \\
\text { nodules }\end{array}$ & Female (\%) & Age (years) & Nodule size $(\mathrm{cm})$ & $\begin{array}{l}\text { Proportion } \\
\text { malignant }^{a}\end{array}$ & Mutations detected \\
\hline Total population & 318 & 362 & $78.7 \%$ & $\begin{array}{l}\text { Range: } 21.8-87.7 \\
\text { Median: } 55.0\end{array}$ & $\begin{array}{l}\text { Range: 1.0-6.6 } \\
\text { Median: } 1.9\end{array}$ & $\begin{array}{l}33 \text { nodules }^{b} \\
(9.1 \%)\end{array}$ & 17 RAS + 9 BRAF + 3 PAX8-PPARY $+{ }^{c}$ \\
\hline $\begin{array}{l}\text { RAS-positive } \\
\text { nodules }\end{array}$ & 17 & 17 & $88.2 \%$ & $\begin{array}{l}\text { Range: } 27.1-63.5 \\
\text { Median: } 46.0\end{array}$ & $\begin{array}{l}\text { Range: } 1.0-5.6 \\
\text { Median: } 1.8\end{array}$ & $\begin{array}{l}8 \text { nodules } \\
(47.1 \%)^{d}\end{array}$ & $\begin{array}{l}8 \text { HRAS+ (3 G12V, } 2 \text { Q61K, } 2 \text { Q61R, } 1 \text { G13R) } \\
7 \text { NRAS+ (6 Q61R, } 1 \text { Q61K) } \\
2 \text { KRAS+ (1 G12V) }\end{array}$ \\
\hline
\end{tabular}

${ }^{\mathrm{a} H i s t o l o g i c a l l y ~ p r o v e n ; ~}{ }^{\mathrm{b}}$ of the 362 nodules, 63 were referred to surgery because of non-benign cytology, of which 33 proved malignant; ${ }^{\mathrm{C}}$ of the three PAX8-PPARY-positive nodules, two underwent surgery and were proven benign. The third nodule underwent Afirma GEC testing, which was also benign; ${ }^{\mathrm{d}}$ of the 17 RAS-positive nodules, ten were referred to surgery because of indeterminate or malignant cytology, of which eight proved malignant. GEC, gene expression classifier 
Table 2 Characteristics of RAS-positive thyroid malignancies

\begin{tabular}{|c|c|c|c|c|c|c|c|c|c|c|}
\hline $\begin{array}{l}\text { Subject } \\
\text { number }\end{array}$ & Sex & Age (years) & RAS mutation & $\begin{array}{l}\text { Nodule size }(\mathrm{mm}) \text { and } \\
\text { parenchyma }\end{array}$ & FNA result & Histopathology & Encapsulated & $\begin{array}{l}\text { Extrathyroidal } \\
\text { extension }\end{array}$ & $\begin{array}{l}\text { Lymph node } \\
\text { metastases }\end{array}$ & $\begin{array}{l}\text { Distant } \\
\text { metastases }\end{array}$ \\
\hline 1 & Female & 27 & HRAS G12V & $\begin{array}{l}10 \times 7 \times 4 \text { Solid No } \\
\text { calcifications }\end{array}$ & Follicular neoplasm & PTC multifocal, $1.2 \mathrm{~cm}$ & $\begin{array}{l}\text { Partially-encapsulated/ } \\
\text { well-circumscribed }\end{array}$ & No & No & No \\
\hline 2 & Female & 46 & HRAS Q61R & $\begin{array}{l}14 \times 9 \times 9 \text { Solid No } \\
\text { calcifications }\end{array}$ & $\begin{array}{l}\text { Suspicious for papillary } \\
\text { carcinoma }\end{array}$ & PTC follicular variant, $1.1 \mathrm{~cm}$ & $\begin{array}{l}\text { Partially-encapsulated/ } \\
\text { well-circumscribed }\end{array}$ & No & No & No \\
\hline 3 & Male & 61 & HRAS Q61R & $\begin{array}{l}36 \times 23 \times 2125-50 \% \\
\text { Cystic No calcifications }\end{array}$ & $\begin{array}{l}\text { Suspicious for papillary } \\
\text { carcinoma }\end{array}$ & PTC follicular variant, $2.6 \mathrm{~cm}$ & $\begin{array}{l}\text { Partially-encapsulated/ } \\
\text { well-circumscribed }\end{array}$ & No & No & No \\
\hline 4 & Female & 33 & HRAS G13R & $\begin{array}{l}20 \times 18 \times 16 \text { Solid No } \\
\text { calcifications }\end{array}$ & $\begin{array}{l}\text { Suspicious for papillary } \\
\text { carcinoma }\end{array}$ & PTC follicular variant, $1.8 \mathrm{~cm}$ & Encapsulated & No & No & No \\
\hline 5 & Female & 44 & NRAS Q61R & $\begin{array}{l}22 \times 11 \times 10 \text { Solid No } \\
\text { calcifications }\end{array}$ & $\begin{array}{l}\text { Malignant - papillary } \\
\text { carcinoma }\end{array}$ & PTC follicular variant, $1.1 \mathrm{~cm}$ & $\begin{array}{l}\text { Partially-encapsulated/ } \\
\text { well-circumscribed }\end{array}$ & No & No & No \\
\hline 6 & Female & 45 & NRAS Q61R & $\begin{array}{l}18 \times 14 \times 7 \text { Solid No } \\
\text { calcifications }\end{array}$ & $\begin{array}{l}\text { Malignant - papillary } \\
\text { carcinoma }\end{array}$ & PTC follicular variant, $1.0 \mathrm{~cm}$ & Encapsulated & No & No & No \\
\hline 7 & Female & 44 & NRAS Q61R & $\begin{array}{l}18 \times 14 \times 12 \text { Solid No } \\
\text { calcifications }\end{array}$ & Follicular neoplasm & PTC follicular variant, $1.6 \mathrm{~cm}$ & Encapsulated & No & No & No \\
\hline 8 & Female & 33 & NRAS Q61R & $\begin{array}{l}31 \times 23 \times 18 \text { Solid No } \\
\text { calcifications }\end{array}$ & $\begin{array}{l}\text { Atypia of undetermined } \\
\text { significance }\end{array}$ & PTC follicular variant, $3.1 \mathrm{~cm}$ & Encapsulated $^{a}$ & No & No & No \\
\hline
\end{tabular}


Table 3 Comparison of RAS-positive and BRAF-positive papillary thyroid cancers

\begin{tabular}{|c|c|c|c|c|c|c|}
\hline Mutation & $\begin{array}{l}\text { Positive predictive value } \\
\text { (test specificity) }\end{array}$ & Tumor size $(\mathrm{cm})$ & Histological subtype & $\begin{array}{l}\text { Lymphovascular } \\
\text { invasion }\end{array}$ & $\begin{array}{l}\text { Extrathyroidal } \\
\text { extension }\end{array}$ & $\begin{array}{l}\text { Lymph node } \\
\text { metastases }\end{array}$ \\
\hline RAS-positive cancers $(\mathrm{n}=8$ ) & $47 \%(97.3 \%)$ & $\begin{array}{l}\text { Range: } 1.0-3.6 \\
\text { Median: } 1.9\end{array}$ & 8 - follicular variant PTC & 0/8 (0 \%) & 0/8 (0 \%) & 0/8 (0 \%) \\
\hline BRAF-positive cancers $\left(\mathrm{n}=8^{\mathrm{a}}\right)$ & $100 \%(100 \%)$ & $\begin{array}{l}\text { Range: } 1.0-4.8 \\
\text { Median: } 1.4\end{array}$ & $\begin{array}{l}6 \text { - classical variant PTC } \\
1 \text { - tall cell variant PTC } \\
1-\text { follicular variant PTC }\end{array}$ & $5 / 8(62.5 \%)$ & $1 / 8(12.5 \%)$ & $1 / 8(12.5 \%)$ \\
\hline
\end{tabular}

${ }^{a}$ Nine BRAF mutations were detected in the cohort. However, one patient did not pursue surgery because of other medical conditions. Therefore eight BRAF-positive cases are shown; bincluding two classical variant PTCs with tall cell features. PTC, papillary thyroid carcinoma

prior ultrasound-guided FNA. We therefore analyzed the repeated sonographic assessment of five $R A S$-positive cytologically benign nodules over time. No significant nodule growth was confirmed in all five $(100 \%)$ cases over a mean duration of 8.3 years (range: $3.1-24.0$ years). Furthermore, no abnormal adenopathy or other sonographically worrisome features were identified throughout follow-up in all patients. For comparison, we randomly selected age and sex matched mutation-negative controls at a 3:1 ratio (i.e., 15 controls) and analyzed the same parameters. Over a comparable mean follow-up time of 7.5 years (range: 2.8-24.0 years), an average $4.6 \mathrm{~mm}$ increase in largest dimension was observed from these cytologically benign, mutation-null nodules.

Four of six RAS-positive, cytologically benign nodules had undergone a separate secondary biopsy at a time point preceding entry into this current study. All four previous aspirates $(100 \%)$ were cytologically benign when performed at mean 5.8 years prior to study entry, confirming no change in cellular morphology to the present. One other nodule demonstrated AUS at cytology on study entry, but was benign on subsequent GEC testing. This nodule was therefore considered benign. Finally, we compared the age of patients with $R A S$ positive thyroid nodules which proved malignant to those which proved benign. Patients with $R A S$-positive benign nodules were on average 13 years older (55.1 (3.7) (mean (SD)) versus $41.9(4.0)$ years; $P=0.028)$ than patients with $R A S$-positive malignant nodules.

\section{Discussion}

Over the last decade, our understanding of the molecular pathways underpinning thyroid cancer has dramatically increased. This has improved care, though simultaneously fostering many clinical assumptions influenced by the population being studied. Nearly all investigations of molecular mutations have been performed on cancerous or cytologically indeterminate thyroid nodules. Importantly, this was not the goal of our study. We sought to present the first blinded, prospective analysis of $R A S$ mutations in a general cohort of patients presenting with nodular disease, to better understand the meaning of such mutations and the natural history of $R A S$-positive nodules. Our data demonstrate a variable and generally low-risk phenotype among most $R A S$-positive nodules. $R A S$-positive status predicts thyroid cancer in $47 \%$ of cases, though is also associated with a large proportion of thyroid nodules with benign histology, benign cytology, and indolent clinical characteristics during long-term conservative follow-up.

In our population, $R A S$-positive thyroid cancers were uniformly low risk - all histologically confirmed to be fvPTC and encapsulated, and all without lymphovascular invasion, extrathyroidal extension, or local lymph node metastases. While many have argued that $R A S$-positive nodules are destined to behave in a malignant fashion, our data suggest this assumption should be viewed with caution. Several of the $R A S$-positive, cytologically benign thyroid nodules in our study cohort had previously been aspirated at an average of 5.8 years prior. This confirms the lack of meaningful cellular transformation over time. Furthermore, highly accurate sonographic assessment of these nodules during a mean 8-year follow-up (and up to 24 years in one patient) confirmed no growth concerning for malignant transformation. Together, these data suggest a far more indolent phenotype inherent to many $R A S$-positive thyroid nodules than previously described.

In the current study, all $R A S$-positive malignancies were fvPTCs (Table 3), while there were no RAS mutations detected in classical variant PTCs. This is interesting in the light of the results of the study of Castro et al., who showed that fvPTCs are molecularly more similar to follicular carcinoma than to classical variant PTCs [22]. This included a higher percentage of RAS mutations in fvPTCs than generally reported in classical variant PTCs.

The ability of activated mutant $R A S$ to induce thyroid neoplasia in vitro has been well established [23-25]. Furthermore, the cellular pathways stimulated by mutated $R A S$, including the MAPK and PI3/AKT signaling pathways, have been linked to thyroid tumorigenesis $[23,26]$. However, the RAS gene encodes a family of three isoforms, NRAS, HRAS, and KRAS, with numerous different mutations described. Some studies have demonstrated a tight association between mutated RAS and follicular thyroid carcinoma, while others confirm a high 
Table 4 Characteristics and sonographic follow-up of RAS-positive benign nodules

\begin{tabular}{|c|c|c|c|c|c|c|c|c|}
\hline $\begin{array}{l}\text { Subject } \\
\text { number }\end{array}$ & Sex & $\begin{array}{l}\text { Age at study } \\
\text { entry (years) }\end{array}$ & $\begin{array}{l}\text { RAS } \\
\text { mutation }\end{array}$ & $\begin{array}{l}\text { Nodule size }(\mathrm{mm}) \text { and } \\
\text { parenchyma at study entry }\end{array}$ & $\begin{array}{l}\text { FNA cytology at } \\
\text { study entry }\end{array}$ & $\begin{array}{l}\text { Previous or subsequent ultrasound } \\
\text { (date, size (mm), parenchyma) }\end{array}$ & $\begin{array}{l}\text { Duration of sonographic } \\
\text { follow-up }\end{array}$ & $\begin{array}{l}\text { Previous FNA cytology } \\
\text { (date, result) }\end{array}$ \\
\hline \multirow[t]{2}{*}{9} & \multirow[t]{2}{*}{ Female } & \multirow[t]{2}{*}{52} & \multirow[t]{2}{*}{ HRAS G12V } & \multirow[t]{2}{*}{$11 / 2010,18 \times 5 \times 11,<25 \%$ Cystic } & \multirow[t]{2}{*}{ Benign } & 02/2013, $16 \times 11 \times 9$, Cystic (<25\%) & \multirow[t]{2}{*}{3.9 years (no growth) } & \multirow[t]{2}{*}{ Not performed } \\
\hline & & & & & & $10 / 2014,17 \times 10 \times 8, N / A$ & & \\
\hline \multirow[t]{3}{*}{10} & \multirow[t]{3}{*}{ Female } & \multirow[t]{3}{*}{52} & \multirow[t]{3}{*}{ HRAS Q61K } & \multirow[t]{3}{*}{ 03/2012, $16 \times 14 \times 9$, Solid } & \multirow[t]{3}{*}{ Benign } & 09/2008, $17 \times 12 \times 11$, Solid & \multirow[t]{3}{*}{3.4 years (no growth) } & \multirow[t]{3}{*}{ 11/2008, Benign cytology } \\
\hline & & & & & & $11 / 2008,17 \times 10 \times 10, N / A$ & & \\
\hline & & & & & & 01/2012, $16 \times 15 \times 9$, Solid & & \\
\hline 11 & Female & 37 & KRAS G12V & $09 / 2011,15 \times 11 \times 9,<25 \%$ Cystic & Benign & - & N/A & Not performed \\
\hline 12 & Female & 54 & KRAS G12V & 10/2010, $14 \times 14 \times 8$, Solid & Benign & $\begin{array}{l}\text { 07/1990, } 13 \times 12 \times 7 \text {, N/A } \\
\text { 10/1993, } 14 \times 12 \times 8 \text {, N/A } \\
05 / 1998,15 \times 13 \times 8 \text {, Solid } \\
06 / 1998,15 \times 13 \times 8 \text {, Solid } \\
\text { 10/1999, } 15 \times 13 \times 7 \text {, Solid } \\
07 / 2003,15 \times 14 \times 7 \text {, Solid } \\
07 / 2005,14 \times 14 \times 8 \text {, Solid } \\
\text { 10/2007, } 15 \times 14 \times 7 \text {, Solid } \\
01 / 2008,13 \times 12 \times 7 \text {, Solid } \\
08 / 2009,14 \times 13 \times 8 \text {, Solid } \\
09 / 2010,16 \times 15 \times 9 \text {, Solid } \\
07 / 2011,16 \times 13 \times 7 \text {, Solid } \\
01 / 2012,15 \times 15 \times 7 \text {, Solid } \\
01 / 2013,16 \times 15 \times 8 \text {, Solid } \\
07 / 2014,15 \times 14 \times 9 \text {, Solid }\end{array}$ & 24.0 years (no growth) & 06/1998, Benign cytology \\
\hline 13 & Female & 54 & NRAS Q61K & $04 / 2011,15 \times 12 \times 11,<25 \%$ Cystic & Benign & $\begin{array}{l}\text { 05/2006, } 15 \times 9 \times 9 \text {, Cystic }(<25 \%) \\
09 / 2006,14 \times 10 \times 9 \text {, Cystic }(<25 \%) \\
06 / 2009,12 \times 11 \times 11 \text {, Cystic }(<25 \%) \\
\text { 04/2011, } 16 \times 13 \times 10 \text {, N/A } \\
\text { 04/2012, } 17 \times 12 \times 12 \text {, Cystic }(<25 \%) \\
06 / 2013,15 \times 12 \times 10, \text { Cystic }(<25 \%)\end{array}$ & 7.0 years (no growth) & 09/2006, Benign cytology \\
\hline 14 & Female & 30 & NRAS Q61R & 09/2011, $16 \times 14 \times 9$, Solid & Benign & $\begin{array}{l}08 / 2008,13 \times 13 \times 8 \text {, Solid } \\
12 / 2008,13 \times 13 \times 9 \text {, Solid } \\
07 / 2011,15 \times 14 \times 8 \text {, N/A }\end{array}$ & 3.1 years (no growth) & 12/2008, Benign cytology \\
\hline \multirow[t]{2}{*}{15} & \multirow[t]{2}{*}{ Female } & \multirow[t]{2}{*}{62} & \multirow[t]{2}{*}{ NRAS Q61R } & \multirow[t]{2}{*}{ 01/2012, $17 \times 14 \times 12$, Solid } & \multirow{2}{*}{$\begin{array}{l}\text { AUS + Afirma GEC } \\
\text { 'Benign' }\end{array}$} & \multirow[t]{2}{*}{ N/A } & \multirow[t]{2}{*}{ N/A } & 04/2012, AUS \\
\hline & & & & & & & & 05/2012, Afirma GEC: 'benign' \\
\hline 16 & Female & 64 & HRAS G12V & $11 / 2010,23 \times 19 \times 15,<25 \%$ Cystic & AUS & N/A & N/A & $\begin{array}{l}\text { 01/2011, Surgery }{ }^{\mathrm{a}} \text { : benign } \\
\text { histology }\end{array}$ \\
\hline 17 & Male & 46 & HRAS Q61K & 03/2012, $56 \times 45 \times 33$, Solid & Follicular neoplasm & N/A & N/A & $\begin{array}{l}\text { 05/2012, Surgery }{ }^{\mathrm{a}} \text { : benign } \\
\text { histology }\end{array}$ \\
\hline
\end{tabular}


prevalence of RAS mutations in benign adenomas or fvPTC [7]. These findings confirm that the MAPK and PI3/AKT cellular pathways are intimately involved in cellular growth and differentiation, but also demonstrate that numerous genetic and epigenetic factors likely contribute to the clinical phenotype. This is exemplified by recent analysis of the thyroid cancer genome atlas [3]. Our data support such findings, while broadening our understanding specific to $R A S$-positivity in a large population, inclusive of mostly benign nodules.

Other published data support our findings. It is again important to note that most investigations have studied $R A S$ mutations only in populations with malignant or indeterminate cytology [2, 27-29]. Similar to us, however, Moses and colleagues performed a blinded and prospective mutational analysis of 417 patients presenting with nodular disease [9]. In this cohort, $21 R A S$-positive nodules were identified ( $4.6 \%$ prevalence), very similar to the $4.7 \%$ detected in our population. While 12 RAS mutations were identified among their 194 patients with abnormal cytology, nine additional $R A S$ mutations were identified in the remaining 257 benign nodules. In total, 6 of 21 (29\%) RAS-positive nodules proved histologically malignant. This rate is comparable to our finding of $47 \%$. Unlike our investigation, however, no previous sonographic analysis or FNA were performed. Nonetheless, these data independently support our conclusion that $R A S$ mutations are commonly detected in benign nodules.

While the number of samples in the current study is too limited from which to draw firm conclusions regarding the distribution of $R A S$ mutation subtypes in benign or malignant nodules, it is noteworthy that two KRAS mutations were detected in benign nodules, while none were detected in malignant nodules. Interestingly, Radkay and colleagues have also studied 204 FNA cases with $R A S$ mutations (mostly indeterminate cytology) with corresponding surgical resection pathological specimens, similarly demonstrating that mutations in KRAS were associated with a significantly lower risk of carcinoma (41.7\%) compared to nodules with HRAS (95.5\%) and NRAS (86.8 \%) mutations [29].

It is unclear why $R A S$-positive thyroid nodules behave in a less virulent manner, though several hypotheses can be considered. One possibility is that the different $R A S$ mutations affect downstream protein function to variable degrees. This hypothesis is supported by parallel evidence observed in medullary thyroid carcinoma patients with activating RET mutations, in which over 50 unique mutations are described [30]. These data confirm that different genetic mutations in the same oncogene lead to variable malignant risk. Separately, it is increasingly likely that a two-hit hypothesis is necessary for malignant transformation in many $R A S$-positive nodules, especially those associated with aggressive disease [31]. Further investigation is required to better address these hypotheses.

Importantly, we do not advocate performing RASmutational testing on all thyroid nodules at time of presentation for several reasons. First, FNA cytology proved more accurate than $R A S$-analysis, as all thyroid malignancies were identified in nodules with indeterminate or malignant cytology. Second, such an approach has not been shown to be cost-effective nor to produce an improved health outcome. We realize, however, that some may nonetheless argue that detection of $R A S$-mutational status is important beyond simply its use in cytologically indeterminate FNAs. RAS-positive nodules indeed carry a higher risk for malignancy compared to $R A S$-negative nodules, and RAS-positive status may also affect prognostic and therapeutic decisions once malignant [8].

Our data do lend preliminary support for surgical removal of $R A S$-positive nodules in younger individuals with non-benign cytology, but question this approach in $R A S$-positive benign nodules. The majority of our study cohort with cytologically benign, yet $R A S$-positive nodules have been closely followed by clinical and sonographic assessment. During a follow-up that ranged from 3-24 years, no significant growth or malignant transformation was documented. Furthermore, repeat aspiration confirmed the presence of consistently benign cytology. Thus, any recommendation for thyroidectomy must balance the presumed benefits of such a procedure against its known risks $[11,12]$.

We acknowledge limitations to our study. Notably, we recruited patients from a single institution. However, the fact that our clinic evaluates $>95 \%$ of all patients seeking thyroid nodule care in our healthcare system improves generalizability of these results and limits selection bias. We similarly acknowledge the lack of universal long-term follow up of all $R A S$-positive nodules. Though our data depict no growth via highly accurate sonographic follow-up of five such patients, it is possible such benign nodules would indeed transform into malignant processes over enough time. Importantly, however, our study also confirmed an impressive low-risk histologic profile to most $R A S$-positive malignancies. Thus, if an observational strategy was followed, repeat sonographic follow-up or repeat FNA may allow the patient and physician to arguably detect any future malignancy while still at a treatable stage. Further prospective study of this hypothesis is required. Finally, one may argue that our study should have been restricted to nodules with indeterminate cytology as this population is in whom mutational testing is currently recommended. However, that was not the goal of our study as we sought to investigate $R A S$-status in all nodules regardless of cytology results. We believe this approach provided 
us the unique opportunity to investigate the natural history and malignant risk of clinically relevant $R A S$ positive nodules in an unbiased fashion.

\section{Conclusions}

In summary, our data depict the blinded, prospective evaluation of $R A S$-mutational status in a population of patients presenting with clinically relevant thyroid nodules, and demonstrate a more indolent and variable phenotype than previously described. $R A S$-positive thyroid nodules, especially in older individuals, frequently demonstrate a benign phenotype. These data therefore support the utility of FNA cytology in guiding the clinical management of $R A S$-positive nodules. Cytologically benign nodules, even if $R A S$-positive, may be candidates for a non-operative observational strategy of repeated sonographic evaluation or FNA. Even if malignant, most $R A S$-positive thyroid nodules appear to be low-risk histologically, and thus are likely to be highly treatable.

\begin{abstract}
Abbreviations
ANOVA: Analysis of variance; AUS: Atypia of undetermined significance; CLIA: Clinical Laboratory Improvement Amendments; FNA: Fine needle aspiration; fvPTC: Follicular variant of papillary thyroid carcinoma; GEC: Gene expression classifier; PTC: Papillary thyroid carcinoma.
\end{abstract}

\section{Competing interests}

EKA has served as a consultant for Veracyte, Inc. DTR and EKA have previously served on the Scientific Advisory Board of Asuragen, Inc. The other authors declare that they have no conflicts of interest. Molecular testing was performed by Asuragen, Inc. as part of a separate research investigation. The company had no role in the design of this study or decision to submit it for publication.

\section{Authors' contributions}

MM and EKA designed and performed the study, analyzed the data, evaluated the results, and drafted and edited the manuscript. NK, TEA, EM, MIK, MCF, CBB, ESC, JAB, JFK, DTR, NLC, AAG, and FDM collected, analyzed and interpreted the data, and edited the manuscript. All authors read and approved the final manuscript.

\section{Acknowledgements}

This analysis and investigation was supported by funding from the Brigham and Women's Hospital and by the NIH T32 DK007529 training grant, and included no industry support. Molecular testing was performed by Asuragen, Inc. as part of a separate research investigation funded to the Brigham and Women's Hospital.

\section{Author details}

${ }^{1}$ Thyroid Section, Division of Endocrinology, Hypertension and Diabetes, The Brigham and Women's Hospital and Harvard Medical School, 75 Francis Street, PBB-B4 Room 417, Boston, MA 02115, USA. ²Department of Pathology, The Brigham and Women's Hospital and Harvard Medical School, Boston, MA, USA. ${ }^{3}$ Department of Radiology, The Brigham and Women's Hospital and Harvard Medical School, Boston, MA, USA. ${ }^{4}$ Department of Surgery, The Brigham and Women's Hospital and Harvard Medical School, Boston, MA, USA.

Received: 5 May 2015 Accepted: 10 July 2015

Published online: 07 August 2015

\section{References}

1. Cantara S, Capezzone M, Marchisotta S, Capuano S, Busonero G, Toti P, et al. Impact of proto-oncogene mutation detection in cytological specimens from thyroid nodules improves the diagnostic accuracy of cytology. J Clin Endocrinol Metab. 2010;95:1365-9.
2. Nikiforov YE, Ohori NP, Hodak SP, Carty SE, LeBeau SO, Ferris RL, et al. Impact of mutational testing on the diagnosis and management of patients with cytologically indeterminate thyroid nodules: a prospective analysis of 1056 FNA samples. J Clin Endocrinol Metab. 2011;96:3390-7.

3. The Cancer Genome Atlas Research Network. Integrated Genomic Characterization of Papillary Thyroid Carcinoma. Cell. 2014;159:676-90.

4. Xing M, Alzahrani AS, Carson KA, Viola D, Elisei R, Bendlova B, et al. Association between BRAF V600E mutation and mortality in patients with papillary thyroid cancer. JAMA. 2013;309:1493-501.

5. Nikiforov YE. Molecular diagnostics of thyroid tumors. Arch Pathol Lab Med. 2011;135:569-77.

6. Xing M, Haugen BR, Schlumberger M. Progress in molecular-based management of differentiated thyroid cancer. Lancet. 2013;381:1058-69.

7. Howell GM, Hodak SP, Yip L. RAS mutations in thyroid cancer. Oncologist. 2013;18:926-32.

8. Ho AL, Grewal RK, Leboeuf R, Sherman EJ, Pfister DG, Deandreis D, et al. Selumetinib-enhanced radioiodine uptake in advanced thyroid cancer. N Engl J Med. 2013;368:623-32

9. Moses W, Weng J, Sansano I, Peng M, Khanafshar E, Ljung BM, et al. Molecular testing for somatic mutations improves the accuracy of thyroid fine-needle aspiration biopsy. World J Surg. 2010:34:2589-94.

10. Rossi M, Buratto M, Tagliati F, Rossi R, Lupo S, Trasforini G, et al. Relevance of BRAFV600E mutation testing versus RAS point mutations and RET/PTC rearrangements evaluation in the diagnosis of thyroid cancer. Thyroid. 2015;25:221-8

11. Bergenfelz A, Jansson S, Kristoffersson A, Martensson H, Reihner E, Wallin G, et al. Complications to thyroid surgery: results as reported in a database from a multicenter audit comprising 3,660 patients. Langenbecks Arch Surg. 2008:393:667-73

12. Sosa JA, Bowman HM, Tielsch JM, Powe NR, Gordon TA, Udelsman R. The importance of surgeon experience for clinical and economic outcomes from thyroidectomy. Ann Surg. 1998;228:320-30.

13. Hay ID, Grant CS, van Heerden JA, Goellner JR, Ebersold JR, Bergstralh EJ. Papillary thyroid microcarcinoma: a study of 535 cases observed in a 50-year period. Surgery. 1992;112:1139-47.

14. Sugino K, Ito Jr K, Ozaki O, Mimura T, Iwasaki H, Ito K. Papillary microcarcinoma of the thyroid. J Endocrinol Invest. 1998;21:445-8.

15. Ito $Y$, Miyauchi A, Inoue $H$, Fukushima M, Kihara M, Higashiyama $T$, et al. An observational trial for papillary thyroid microcarcinoma in Japanese patients. World J Surg. 2010;34:28-35.

16. American Thyroid Association Guidelines Taskforce on Thyroid Nodules and Differentiated Thyroid Cancer, Cooper DS, Doherty GM, Haugen BR, Kloos RT, Lee SL, et al. Revised American Thyroid Association management guidelines for patients with thyroid nodules and differentiated thyroid cancer. Thyroid. 2009:19:1167-214.

17. Gharib H, Papini E, Paschke R, Duick DS, Valcavi R, Hegedüs L, et al. AACE/ AME/ETA Task Force on Thyroid Nodules. American Association of Clinical Endocrinologists, Associazione Medici Endocrinologi, and European Thyroid Association medical guidelines for clinical practice for the diagnosis and management of thyroid nodules: executive summary of recommendations. J Endocrinol Invest. 2010;33:287-91.

18. Beaudenon-Huibregtse S, Alexander EK, Guttler RB, Hershman JM, Babu V, Blevins TC, et al. Centralized molecular testing for oncogenic gene mutations complements the local cytopathologic diagnosis of thyroid nodules. Thyroid. 2014:24:1479-87.

19. Cibas ES, Ali SZ. NCI Thyroid FNA State of the Science Conference. The Bethesda system for reporting thyroid cytopathology. Am J Clin Pathol. 2009;132:658-65.

20. Alexander EK, Kennedy GC, Baloch ZW, Cibas ES, Chudova D, Diggans J, et al. Preoperative diagnosis of benign thyroid nodules with indeterminate cytology. N Engl J Med. 2012;367:705-15.

21. Yassa L, Cibas ES, Benson CB, Frates MC, Doubilet PM, Gawande AA, et al. Long-term assessment of a multidisciplinary approach to thyroid nodule diagnostic evaluation. Cancer. 2007;111:508-16.

22. Castro P, Rebocho AP, Soares RJ, Magalhaes J, Roque L, Trovisco V, et al. PAX8-PPARY rearrangement is frequently detected in the follicular variant of papillary thyroid carcinoma. J Clin Endocrinol Metab. 2006:91:213-20.

23. Nikiforov YE, Nikiforova MN. Molecular genetics and diagnosis of thyroid cancer. Nat Rev Endocrinol. 2011;7:569-80.

24. Pai EF, Kabsch W, Krengel U, Holmes KC, John J, Wittinghofer A. Structure of the guanine-nucleotide-binding domain of the Ha-ras oncogene product p21 in the triphosphate conformation. Nature. 1989;341:209-14. 
25. Suarez HG, Du Villard JA, Caillou B, Schlumberger M, Tubiana M, Parmentier C, et al. Detection of activated ras oncogenes in human thyroid carcinomas. Oncogene. 1988;2:403-6.

26. Xing M. Genetic alterations in the phosphatidylinositol-3 kinase/Akt pathway in thyroid cancer. Thyroid. 2010;20:697-706.

27. Gupta N, Dasyam AK, Carty SE, Nikiforova MN, Ohori NP, Armstrong M, et al. RAS mutations in thyroid FNA specimens are highly predictive of predominantly low-risk follicular-pattern cancers. J Clin Endocrinol Metab. 2013;98:E914-22.

28. Nikiforov YE, Carty SE, Chiosea SI, Coyne C, Duvvuri U, Ferris RL, et al. Highly accurate diagnosis of cancer in thyroid nodules with follicular neoplasm/ suspicious for a follicular neoplasm cytology by ThyroSeq v2 nextgeneration sequencing assay. Cancer. 2014;120:3627-34.

29. Radkay LA, Chiosea SI, Seethala RR, Hodak SP, LeBeau SO, Yip L, et al. Thyroid nodules with KRAS mutations are different from nodules with NRAS and HRAS mutations with regard to cytopathologic and histopathologic outcome characteristics. Cancer Cytopathol. 2014;122:873-82.

30. Krampitz GW, Norton JA. RET gene mutations (genotype and phenotype) of multiple endocrine neoplasia type 2 and familial medullary thyroid carcinoma. Cancer. 2014;120:1920-31.

31. Nikiforova MN, Wald Al, Roy S, Durso MB, Nikiforov YE. Targeted nextgeneration sequencing panel (ThyroSeq) for detection of mutations in thyroid cancer. J Clin Endocrinol Metab. 2013;98:E1852-60.

\section{Submit your next manuscript to BioMed Central and take full advantage of:}

- Convenient online submission

- Thorough peer review

- No space constraints or color figure charges

- Immediate publication on acceptance

- Inclusion in PubMed, CAS, Scopus and Google Scholar

- Research which is freely available for redistribution 